\title{
Design of Temperature Control System Based on Vacuum Furnace
}

\author{
Gehua Chen a , Dongdong Song \\ School of electrical \&Electronic Engineering, Changchun University of Technology, Changchun \\ 130012, China. \\ a1983827455@qq.com
}

Keywords: Vacuum furnace; Single channel single light path and double color ratio temperature measurement; Intelligent control temperature; Continuous temperature measurement.

\begin{abstract}
The importance of continuous temperature measurement in vacuum furnace to realize the temperature control of vacuum furnace is expounded by the universality of the vacuum furnace application and the control of the quality and process of the applied products. This paper briefly introduces the traditional method of high temperature measurement and control, compares the advantages of the single channel single channel double color ratio measurement method, and the temperature control scheme of the vacuum furnace using Smith intelligent control algorithm. It can effectively reduce the error of the temperature collection system, and make the end hit rate of the measurement of the temperature of different metals 3\%. In addition, accurate control of vacuum furnace temperature is achieved.
\end{abstract}

\section{Introduction}

In modern technology, the vacuum heat treatment process has been widely applied to the steel manufacturing industry. Because of the particularity of the vacuum environment, it is impossible for people to measure the heat transfer between the workpiece in the vacuum heat treatment and the complex heat transfer between the surrounding medium. In the traditional temperature measurement and control of the vacuum heat treatment, most of the engineers adopt the experience estimation method and the thermocouple, and the cost of this kind of method is high, and the huge manpower and material resources are wasted. At present, this traditional method of research cannot meet the requirements of the development of modern manufacturing industry. Backward design ideas and methods have gradually become the bottleneck restricting the rapid development of modern manufacturing industry.

The temperature of the vacuum furnace in the field cannot be quickly heated to the target temperature. The fuzzy Smith intelligent control algorithm is a time delay prognosis compensation algorithm, which can solve the time delay problem in the temperature control system.

In order to meet the needs of the intelligent manufacturing industry, an intelligent instrument for real-time and accurate measurement and control of vacuum furnace temperature is urgently needed. In this paper, an intelligent instrument for measuring and controlling furnace temperature is designed to meet this trend.

\section{Measuring Part}

\subsection{Field Map}

The overall diagram of the experimental site is shown in Fig. 1. (a), the heating furnace diagram in vacuum furnace is shown in Fig. 2. (b), and the thermometer being measured is shown in Fig. 3. (c). 


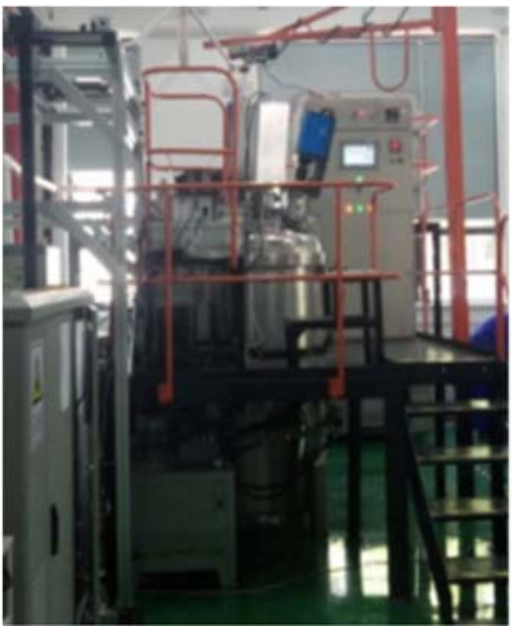

(a)

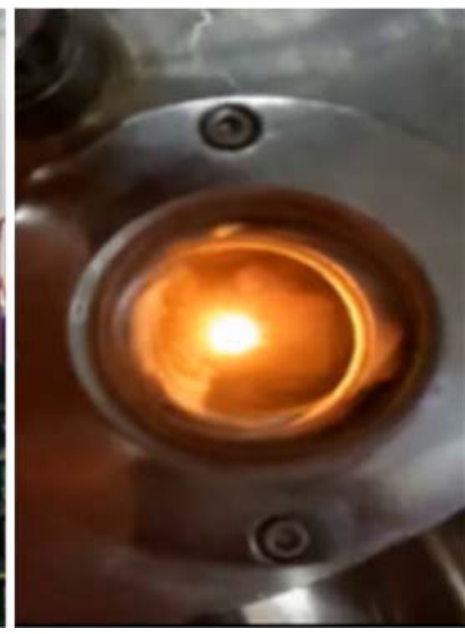

(b)

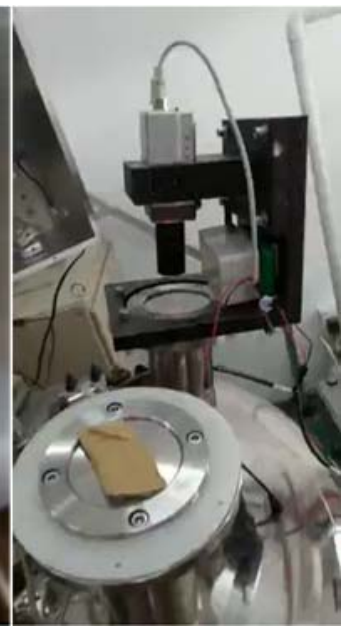

(c)

Figure 1. Field test picture

\subsection{Measuring Principle}

Compared with the traditional method of measuring temperature of contact thermocouple, the single channel single light path two color ratio temperature measurement method is used in this paper, and the non-contact method is used to measure temperature through the perspective window. The temperature is measured by comparing the wavelengths of infrared rays at different wavelengths of the molten steel in a vacuum furnace, which is greatly transformed in the structure of the software and the hardware relative to the previous infrared high temperature measurement. The experiment has also achieved good results.

The method of measuring temperature by dual color ratio is to measure the temperature by measuring the ratio of spectral radiance of thermal radiator at two or more than two wavelengths. It has high accuracy, quick response and small target observation (the minimum can reach $2 \mathrm{~mm}$ ). The monochromatic blackness coefficient of the actual object $\varepsilon \lambda$ Tand Total radiation blackening coefficient $\varepsilon \mathrm{T}$ is difference. However, the ratio of monochromatic coefficient $\varepsilon \lambda 1$ TandE $2 \mathrm{~T}$ for different wavelengths of the same object is very small. So, the temperature measured by the double color ratio thermometer is called the colorimetric temperature $\mathrm{TS}$, which is very close to the real temperature $\mathrm{T}$ of the object, and generally cannot be corrected.

By the ven theorem (1), it is known that when the blackbody temperature changes, the maximum radiant ejection will move in the direction of increasing or decreasing the wavelength, which will change the brightness at the specified two wavelengths at $\lambda 1$ and $\lambda 2$, and the corresponding temperature values can be obtained by measuring the ratio.

$$
M_{0}(\lambda, T)=C_{1} \lambda^{-5} e^{-\frac{C_{2}}{\lambda T}}
$$

For a blackbody with a temperature of $\mathrm{T}$, the ratio of spectral radiance at wavelengths of lambda $\lambda 1$ and lambda $\lambda 2$ is $\mathrm{R}$, according to the Wien theorem.

$$
\mathrm{R}=\frac{L_{\lambda 1}^{0}}{L_{\lambda 2}^{0}}=\left(\frac{\lambda 2}{\lambda 1}\right)^{5} \cdot e^{\frac{C 2}{T}\left(\frac{1}{\lambda 2}-\frac{1}{\lambda 1}\right)}
$$

After the logarithm is taken

$$
\ln \mathrm{R}=\ln \frac{L_{\lambda 1}^{0}}{L_{\lambda 2}^{0}}=5 \ln \left(\frac{\lambda 2}{\lambda 1}\right)+\frac{C 2}{T}\left(\frac{1}{\lambda 2}-\frac{1}{\lambda 1}\right)
$$

The upper form can be simplified to

$$
\ln R=A+B T^{-1}
$$

In the formula, $\mathrm{A}=5 \ln \left(\frac{\lambda_{1}}{\lambda_{2}}\right), \mathrm{B}=C_{2}\left(\frac{1}{\lambda_{2}}-\frac{1}{\lambda_{1}}\right)$

It can be seen from the above formula that the formula (3) can be obtained. 


$$
T=\frac{C_{2}\left(\frac{1}{\lambda_{2}}-\frac{1}{\lambda_{1}}\right)}{\ln R-5 \ln \frac{\lambda_{2}}{\lambda_{1}}}
$$

According to the definition of Colorimetric Thermometer, the relationship between the real temperature of the object and the colorimetric temperature can be further obtained.as follows:

$$
\frac{1}{\mathrm{~T}}-\frac{1}{T_{s}}=\frac{\ln \frac{\varepsilon_{\lambda 1 T}}{\varepsilon_{\lambda 2 T}}}{C_{2}\left(\frac{1}{\lambda 1}-\frac{1}{\lambda 2}\right)}
$$

In the form, $\varepsilon \lambda 1 \mathrm{~T}$ and $\varepsilon \lambda 2 \mathrm{~T}$ are the monochromatic coefficients of objects at $\lambda 1$ and $\lambda 2$ respectively. $\mathrm{T}$ is the real temperature of the object, and TS is the colorimetric temperature of the object.

The schematic diagram of the thermometer is shown in Fig. 2. The method of measuring the vacuum furnace adopts the single channel single optical path double color ratio temperature measurement method. Effective elimination of external dust, temperature and other environmental interference factors, the measured data through fuzzy control algorithm can be more accurate. The transmitting part of the data is transmitted by infrared mode. The power supply part adopts wireless power supply, and the rotary motor adopts brushless DC motor.

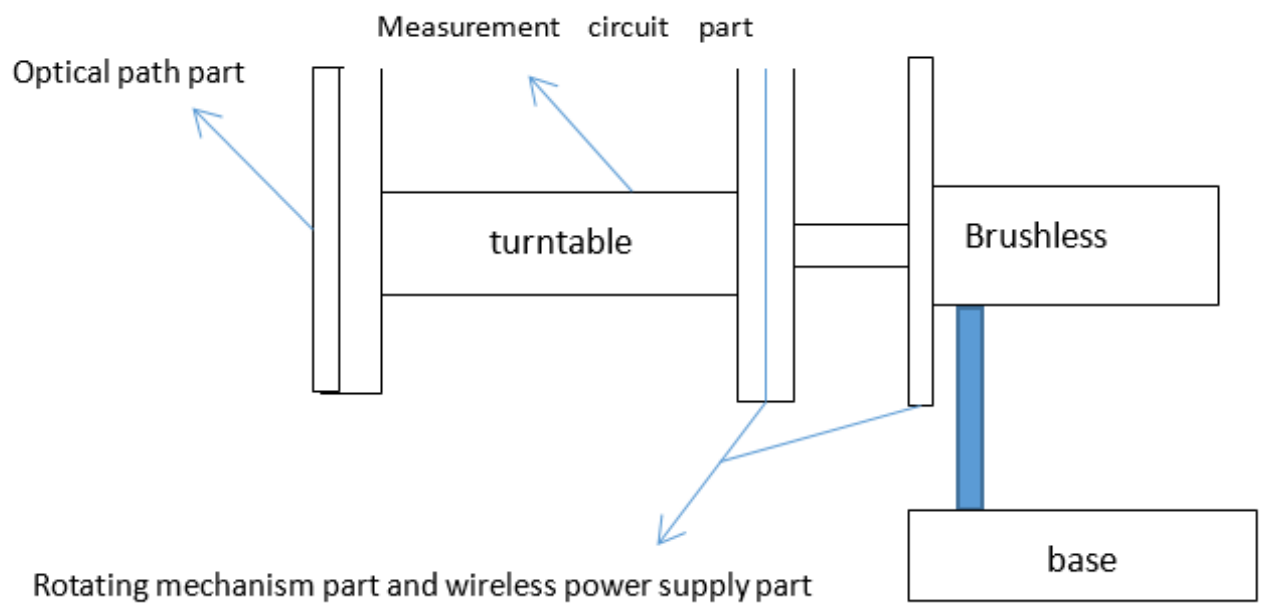

Figure 2. Schematic diagram of thermometer structure

\subsection{The Establishment of a Temperature Measuring Compensator}

The fuzzy algorithm is used in the thermometer of this paper. The block diagram of fuzzy controller is shown in Figure 3. It consists of three parts: fuzzification, fuzzy reasoning and nonfuzzification, and the three is based on knowledge base. First, according to the actual temperature measured by the thermocouple in vacuum furnace as the standard temperature, it is used as the basis for building knowledge base. The measured value of the thermometer is taken as input, and is fuzzed, fuzzy and defuzzification.

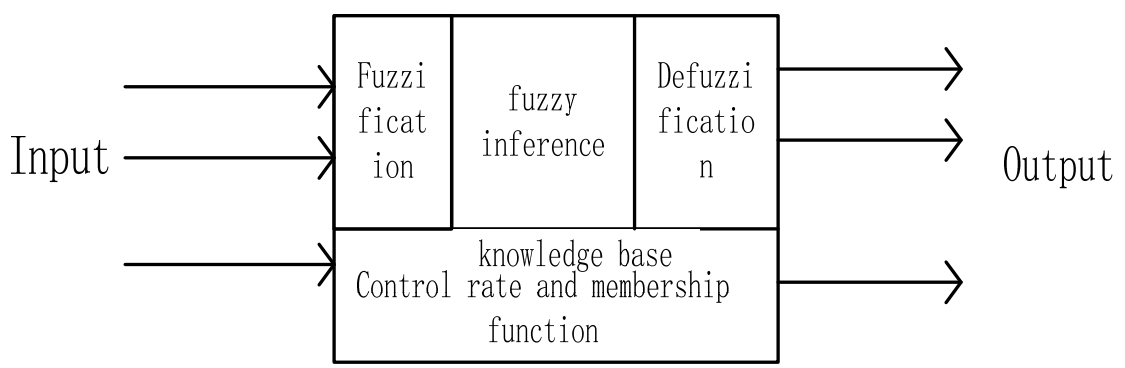

Figure 3. A schematic diagram of fuzzy algorithm

The temperature measuring flow chart of the thermometer is like Figure 4. 


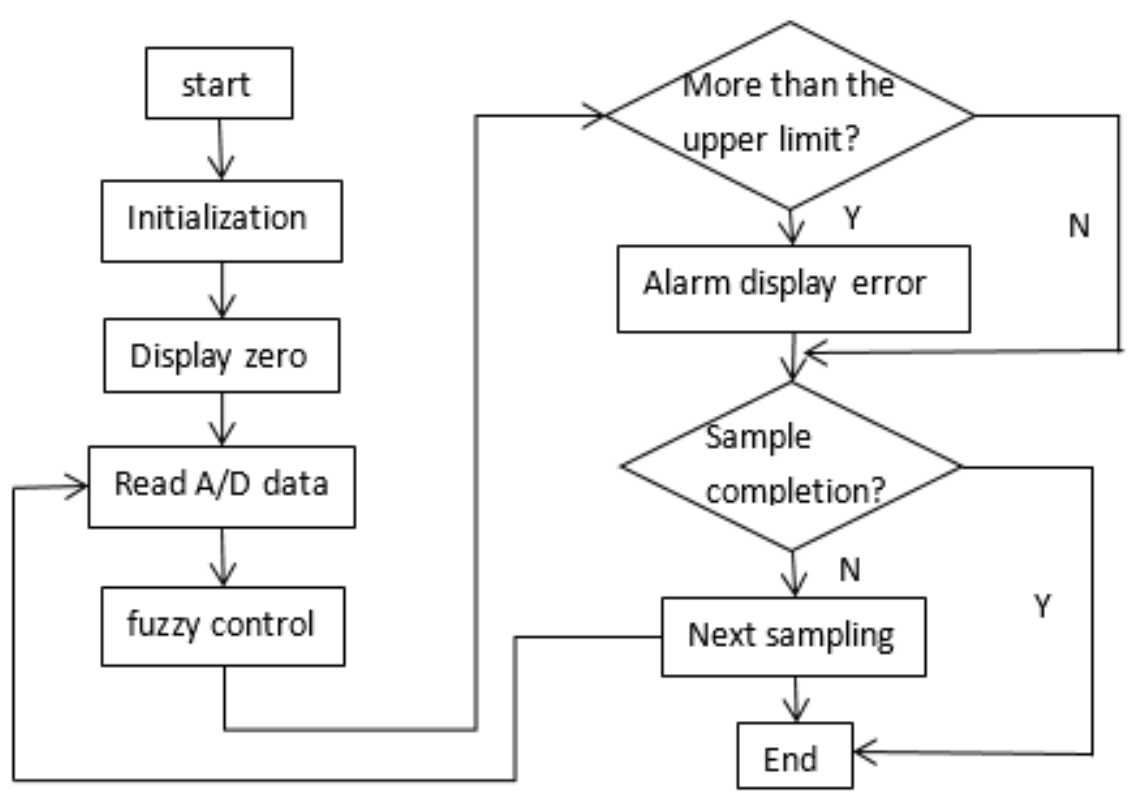

Figure 4. Flow chart of measuring and realizing thermometer

\section{Control Section}

A schematic diagram of the overall temperature control in vacuum furnace is shown in Fig. 5:The temperature actual value of the vacuum furnace is sent to the MCU end by the thermometer, and the corresponding control amount is calculated by the Smith intelligent control algorithm written by MCU, then the $\mathrm{D} / \mathrm{A}$ converter is transformed into the current, and the conduction angle of the thyristor is controlled by the trigger, thus the current size in the vacuum wire is changed and the purpose of controlling the furnace temperature is achieved.
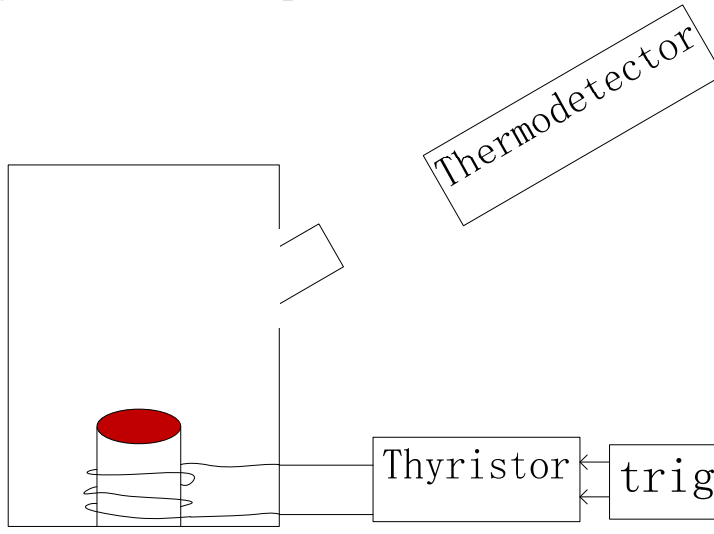

Figure 5. Schematic diagram of temperature control structure

Through the temperature measured by the thermometer, the measured temperature is transferred to the temperature controller of the furnace body of the vacuum furnace, because the time delay and the pure hysteresis are the most common factors affecting the control system in the industrial production. In the vacuum furnace temperature control system, the existence of time delay and pure hysteresis not only affects the temperature control accuracy of the vacuum furnace, but also affects its working efficiency and product yield. The principle of temperature control with pure hysteresis is shown in Figure 6. 


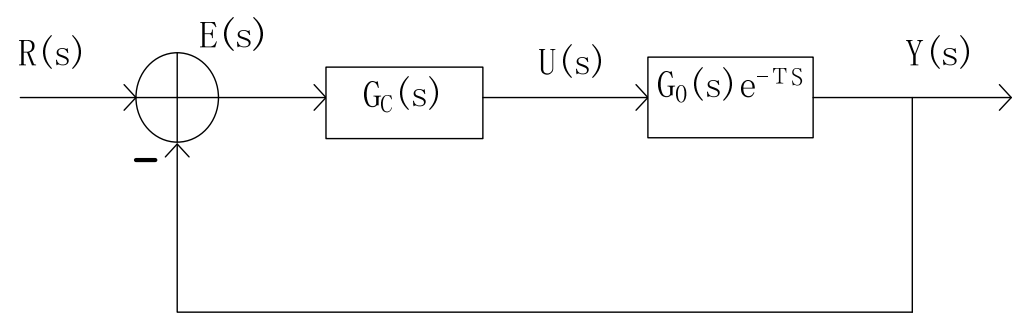

Figure 6. Temperature control with pure lag

The Gc (s) in the diagram is a temperature-controlled transfer function. $\mathrm{G}_{0}(\mathrm{~s}) \mathrm{e}^{-\tau \mathrm{s}}$ or the transfer function of the controlled object, the partial transfer function without pure delay is G0 (s), while the transfer function with pure delay part is $\mathrm{e}^{-\tau \mathrm{s}}$.

According to the analysis of closed loop transfer function of vacuum furnace temperature control, the function can be identified as:

$$
\Phi(s)=Y(s) / R(s)=\left(G_{c}(s) \times G_{0}(s) e^{-\tau s}\right) \div\left(1+G_{c}(s) \times G_{0}(s) e^{-\imath s}\right)
$$

The characteristic equation of closed loop transfer function of vacuum furnace temperature control system is:

$$
1+\left(G_{c}(s) \times G_{0}(s) e^{-\tau s}\right)=0
$$

From the formula (8), it is known that in the closed loop traditional function characteristic equation of the temperature control system, the $\mathrm{e}^{-\tau s}$ terms of the transfer function with the pure lag part, that is, the pure lag link of the system, affect the stability of the temperature control system of the vacuum furnace.

According to the Smith prestimation compensation control method proposed by O. J. Smith, for example, after the temperature control transfer function and the $\mathrm{N}$ determination treatment before the pure lag remission, the ideal temperature control system will be formed, and the temperature control transfer function of the vacuum furnace under the ideal state is as follows:

$$
\Phi(s)=Y(s) / R(s)=\left(G_{c}(s) \times G_{0}(s) e^{-\tau s}\right) \div\left(1+G_{c}(s) \times G_{0}(s)\right)
$$

The characteristic equation of closed loop transfer function of vacuum furnace temperature control system under ideal condition is:

$$
1+\mathrm{G}_{\mathrm{c}}(s) \times G_{0}(s)=0
$$

At this time, the G0 (s) output signal in the temperature control system is the feedback signal of the system, the vacuum furnace temperature control system under the ideal state, the G0 (s) feedback signal is ahead of the time of tau. According to formula (4), after controlling the vacuum furnace temperature control system, the control quality of the system has been greatly improved. Therefore, in the process of the actual vacuum furnace temperature control system, the Smith prediction compensation control system will be introduced to obtain the ideal control state after the transfer of the pure hysteresis loop of the vacuum furnace temperature control system, to improve the control quality of the temperature control system and to accelerate the response time of the temperature control system.

\section{Experimental Analysis:}

The optical path is an important factor affecting the temperature accuracy of the thermometer. The experiment adopts a single optical path, and the motor's no-load speed is $5730 \mathrm{r} / \mathrm{min}$. The distance is also fixed in $1 \mathrm{~cm}$ because of the wireless charging and distance. Figure 7 is the data that is not checked before the temperature measurement. 


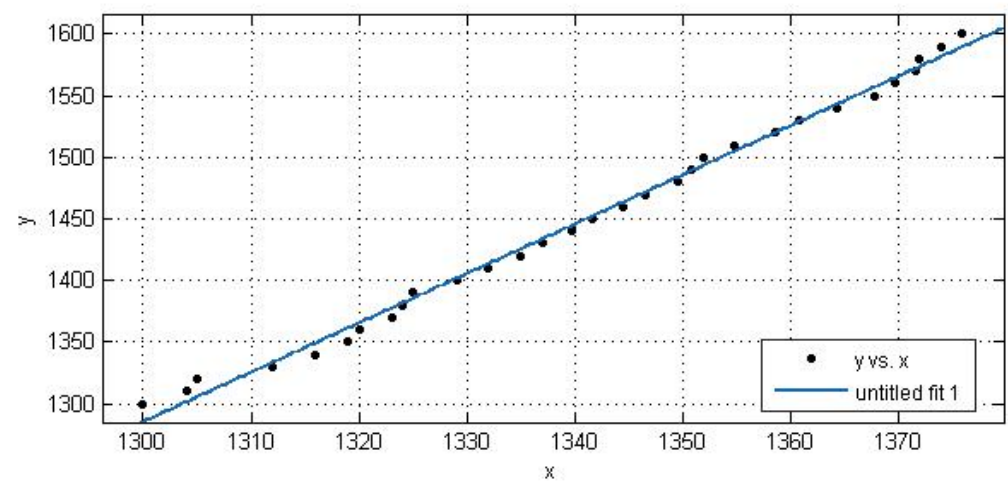

Figure 7. Trend map of uncorrected pre-measurement data

From the curve of the graph, it can be seen that the monotonicity of the function obtained by the thermometer can be monotonous, but the real time accuracy of the data is very poor, and the temperature of the vacuum furnace cannot be reacted in real time. The measured temperature data are processed by fuzzy control algorithm, and the data obtained are shown in Figure 8.

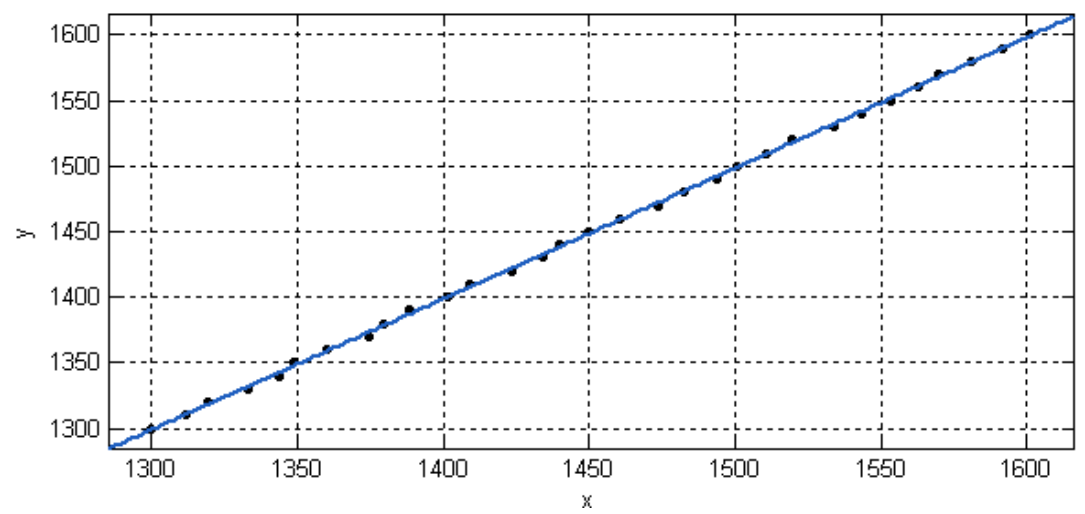

Figure 8. Trend map of revised measurement data

The final hit rate of the measured data is less than $3 \%$, and the temperature of the metal in the vacuum furnace can be measured in real time. On the basis of the accurate measurement of the temperature of the metal in the furnace, the data is transmitted to the temperature controller of the vacuum furnace in real time, and the Smith compensation control system is used. The temperature of the heating furnace of the vacuum furnace can reach the specified temperature quickly, as shown in Table 1.

Table 1. Temperature and time of vacuum furnace

\begin{tabular}{|c|c|c|c|c|c|c|c|}
\hline Time $(\mathrm{S})$ & 1440 & 1740 & 1980 & 2280 & 2460 & 2940 & 3300 \\
\hline Temperature $\left({ }^{\circ} \mathrm{C}\right)$ & 1300 & 1400 & 1500 & 1600 & 1700 & 1800 & 1800 \\
\hline
\end{tabular}

\section{Conclusion}

After the analysis of the experimental data and the actual experimental results, the measured temperature can be displayed more accurately and quickly through the comparison and analysis with the thermometer without the algorithm. The proposed method only effectively improves the error of the measurement of the optical path and makes some optimization in the circuit. At the same time, the technology has a major breakthrough in the practical aspect, the shape of the processed product and the improved space. If the improved technology is combined with the design, it will make it possible to do it. The products that come out can be widely used.

\section{Acknowledgments}

The research content of this paper comes from the "on-line infrared monitoring system of melt temperature in smelting furnace" (project number: 20180201019GX), which is the key project of the science and Technology Department of Jilin province. Thank you very much for your guidance and help. Teachers and classmates. 


\section{References}

[1]. Ge-hua Chen, Qing-hua Yao, De-jiang Zhang. Detection and analysis technology of on-line temperature and element content in AOD furnace2012,2(45-95).

[2]. Hui-ran Li On line detection method of ferroalloy furnace temperature in argon oxygen refining Changchun University of Technology 2010.3.

[3]. Shao-bo Li Application of cryogenic pump in ultra-high vacuum furnace Beijing Vacuum Electronic Technology Corporation 2017.2.

[4]. Juan JIMENEZ, Javier MOCHON, Jesus Sainz de AYALA. Blast furnace hot metal temperature prediction through neural networks-based models. ISIJ International,2004,44(3):573-580.

[5]. W.B. Spillman, Jr. Multimode. fiber-optical pressure sensor based on the photo elastic effect.Opt.Lett.,2002,7(8):388-390.

[6]. Bin Meng. Discussion on the application of vacuum furnace in tin refining process. Laibin China tin smelting Co., Ltd.,2016.11.

[7]. Xin-yan Chen. Design of circulating cooling water system in vacuum furnace. Industrial Technology,2016.10.054.

[8]. Loesener, Neuer, G. New far-infrared pyrometer for radiation temperature measurement on semitransparent. Measurement: Journal of the International Measurement Confederation . 1994.

[9]. Jie Liu, Xi-nan Zhang. Analysis and measures of defects in vacuum heat treatment furnace. Heat treatment technology and equipment. 2014.10.

[10]. Kui-han Zhang, Ming-xu Huang. Special thermocouple in vacuum furnace and its selection. 2014.11.

[11]. Wang J, Jiang-Hui D I, Yue W U, et al. Infrared colorimetric temperature measurement for AOD furnace[J]. Journal of Changchun University of Technology, 2014.

[12]. Guo F T, Liang X U, You W. Splash forecasting of AOD furnace based on a multi-sensor information fusion algorithm[J]. Journal of Changchun University of Technology, 2014. 